\section{Acute epididymo-orchitis complicating Streptococcus pneumoniae bacteremia in an adult smoker and alcoholic with community acquired pneumonia}

\section{Ibrahim TM, ${ }^{*}$ Letchumanan MN}

General and Acute Medicine Department Goulbourn Valley Base Hospital Graham Street, Shepparton, Vic 3630, Australia

\begin{abstract}
INTRODUCTION: Although hematogenous acute epididymo-orchitis complicating Gram negative bacteremia, tuberculosis and brucellosis have been reported in medical literature, there is paucity of report on acute epididymo-orchitis complicating Streptococcus pneumoniae bacteremia.
\end{abstract}

CASE REPORT: We report the case of hematogenous acute epididymo-orchitis from $S$. pneumoniae bacteremia in an adult chronic cigarette smoker and alcoholic with community acquired pneumonia.

CONCLUSION: We recommend that acute epididymo-orchitis should be remember as a sequel of $S$. pneumoniae bacteremia especially in adult males with immune suppression

KEY WORDS: Acute epididymo-orchitis, Streptococcus pneumoniae, Community acquired pneumonia

Article submitted 23 November. Reviewed 12 December. Author correction 21 Decembert. Final version accepted 31 December 2012

*Correspondence to: Dr. Tunde Maiyaki Ibrahim, General and Acute Medicine Department Goulbourn Valley Base Hospital Graham Street, Shepparton, Vic 3630, Australia, email: tuned.ibrahim@gvhealth.org.au, Tel. No.: (+61)0358322322 / 04023520284 


\section{INTRODUCTION}

Extra pulmonary complications associated with Streptococcus pneumonieae bacteremia from community acquired pneumonia (CAP) are well known and this include some serious conditions such as meningitis, brain abscess, purulent pericarditis, endocarditis, osteomyelitis, and septic arthritis, but acute epididymo-orchitis from $S$. pneumoniae bacteremia was rarely reported in medical literature. ${ }^{1-3}$ We report this rare case of acute epididymo-orchitis in an adult with $S$. peumoniae bacteremia complicating CAP to draw clinicians attention to this rare association especially in immune compromised adults.

\section{CASE REPORT}

We are reporting a case of acute epididymo-orchitis associated with $S$. pneumoniae bacteremia from CAP. A 50 yr old man chronic cigarette smoker with alcohol use disorder presented with 5 days of productive cough, fever, chills and rigors. $\mathrm{He}$ started to experience right testicular pain and subsequently scrotal swelling 2 days later. But no urethral discharge nor dysuria and his last sexual contact was approximately a year back. He had testicular torsion at age 12 and underwent bilateral orchidopexy. On physical examination he had systemic inflammatory response signs of fever with body temperature of $38.9{ }^{\circ} \mathrm{C}$, tachycardia (HR of $118 \mathrm{bpm}), \mathrm{BP}, 110 / 40 \mathrm{mmHg}$ and low oxygen saturation of $92 \%$ on ambient air. Chest examination revealed evidence of right middle lobe consolidation and scrotal examination revealed inflamed right scrotum with tender swollen right testicle. Laboratory investigations revealed elevated bio-inflammatory makers (CRP $=136 \mu \mathrm{g} / \mathrm{l}$, WCC $=22.8 / \mu \mathrm{L}, \quad$ Neutrophil $=19.4 / \mu \mathrm{l}), \quad$ LFT was essentially normal except for low synthetic function with albumin of $25 \mathrm{gm} / \mathrm{l}$, the patient's blood cultures yielded $S$. pneumoniae in horse blood agar confirm to be serotype 20 by enzyme-link immunosorbent assay (ELISA) test. The $S$. pneumoniae was sensitive to peniciline, ceftriaxone and vancomycin. The urine microscope and culture was negative. The CXR showed right middle lobe consolidation and the scrotal ultrasound showed swollen and inflamed right epididymis and testicle (Figure 1). He was treated with ceftriaxone, roxithromycine, and ibuprofen empirically for 48 hrs and on confirmation of the isolate from blood culture roxithromycine was dropped and ceftriaxone continued for another 3 days. His respiratory and scrotal symptoms and the bioinflammatory markers significantly improved and
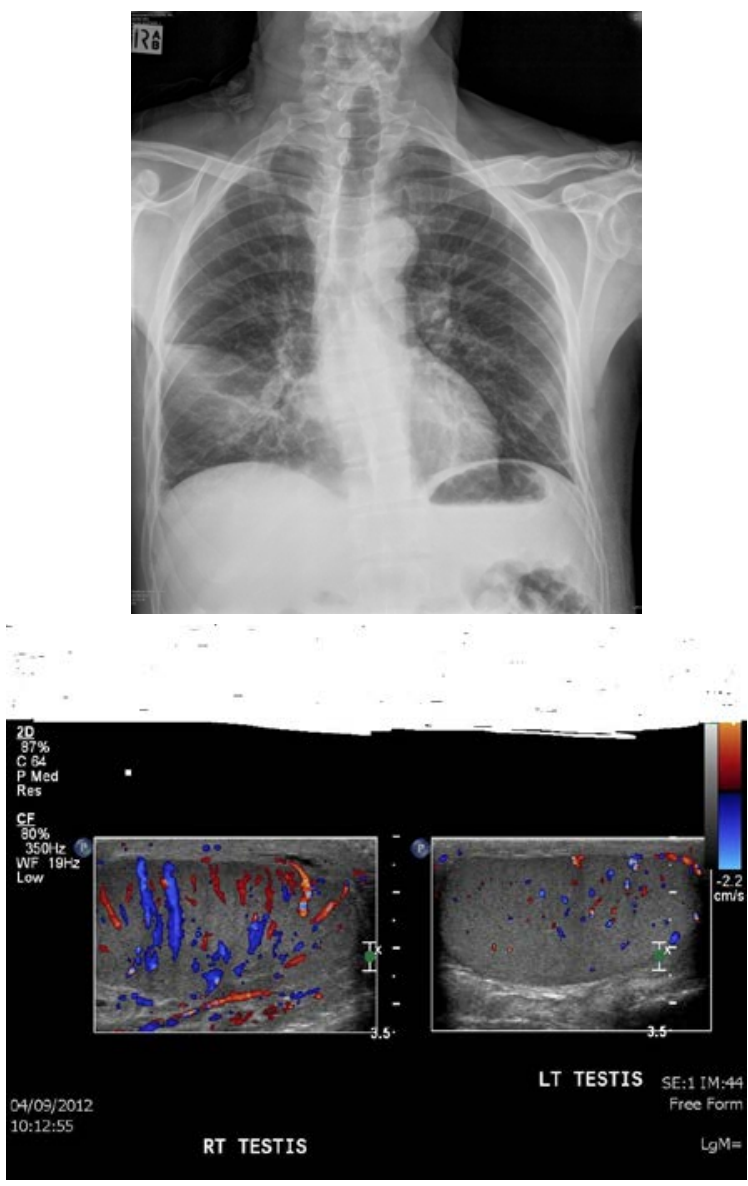

Figure 1: Chest X-ray of the patient showing right middle lobe consolidation (above) and ultrasound of the testicles showing hypervascularity of the right testis (below).

was discharged on day 5 on amoxicillin/clavulanate for another 5 days.

\section{DISCUSSION}

The common causes of acute epididymo-orchitis in adults include Chlamydia trachomatis, Neisseria gonorrhoeae and the Enterobactericea. The pathogenesis except in mumps commonly involves retrograde spread of these organisms from the urinary tract during the cause of urinary tract infection (UTI) or sexually transmitted diseases(STD).4-6 Hematogenous spread of microorganisms to the epididymis and testicles can occur in adults $>30$ years during bacteremia, viremia and septicemia to cause inflammation and infections resulting in epididymo-orchitis. Even though rare this as been reported in Gram negative bacteriemia (Salmonella and Pseudomonas spp.), mumps, 
tuberculosis and brucellosis. ${ }^{7-10}$ Hematogenous spread of $S$. pneumoniae resulting in acute epididymo-orchitis to our knowledge was rarely found in medical literature. Apart from the S. pneumoniae bacteremia from the community acquired pneumonia (CAP) our patient had no other reason for his epididymo-orchitis. The risk factors for his severe invasive pneumococcal infection could be his significant cigarette smoking and alcohol abuse both of which are causes of local respiratory tract and systemic immune suppression which significantly increases the risk of invasive pneumococcal infection. ${ }^{3,11}$

The common clinical presentations of acute epididymo-orchitis include acute scrotal pain and swelling which may be associated with systemic symptoms such as fever and sweating. Our patient had all of these classical features. However symptoms of underlining UTI or STD like dysuria, pelvic discomfort and urethral discharge which are common features in retrograde epididymo-orchitis were absent in our patient. ${ }^{4,5}$

The screening investigations usually include urinary and urethral discharge microscope and culture, urethral discharge PCR for Chlamydia and Gonococcus spp., blood culture, full blood count, CRP and ESR. ${ }^{6}$ Tuberculosis and brucellosis screening may be indicated in endemic regions when the causative organism is not readily obvious. As expected in most cases of infections with bacteremia the bio-inflammatory markers were elevated in our patients and the blood cultures grew $S$. pneumoniae .While the urine culture was none remarkable. Diagnostic scrotal ultrasound with or without needle aspiration biopsy is the best imaging modality to diagnose epididymo-orchitis and rule out other causes of acute scrotal pain and swelling such as testicular infarct from torsion, abscess,infected hydrocele and rarely malignancies and hematoma. As demonstrated in our patient testicular ultrasound in epididymo-orchitis shows swelling and increase vascularity and in some circumstances abscess formation in the epididymis and the testicle. In rare circumstances testicular margnet resonance imaging with or without needle aspiration biopsy may be indicated. ${ }^{12-14}$

Our patient was successfully treated with initial ceftriaxone and subsequently amoxicillin/clavulate combination both of which the $S$. pneumoniae was sensitive. The empiric antibiotic recommended by the British Association for Sexual Health and HIV (BASHH) UK guideline for the management of epididymo-orchitis, 2010 include ceftriaxone and doxycycline. $^{15}$ When coliform bacteria are suspected, ofloxacin or levofloxacin is recommended, but the definitive treatment would however be guided by the culture and antibiotic sensitivity report as was the case in our patient. ${ }^{6}$ Delavierre, ${ }^{5}$ in France also reported that adults less than 35 years of age with urethritis and suspected STD, can be treated with tetracyclines as both $C$. trachomatis and N. gonorrhoeae are sensitive to it. $N$. gonorrheae and C. trachomatis also responds to other antimicrobials, such as ceftriaxone, macrolides and second-generation quinolones. Typically, treatment is given for 3 weeks. Our patient was however treated for 2 weeks with excellent outcome. In cases associated with STD sexual partners should be evaluated and treated to prevent reinfection. In patients older than 35 years who have positive urine cultures for bacteria, urinary tract symptoms, a prior diagnosis of a urinary tract abnormality, or a history of a recent transurethral procedure, treatment with extra strength cotrimoxazole, quinolones, aminoglycoside or cephalosporin can be used. 5,6

If inadequately managed or not recognized in time acute epididymo-orchitis can result in epididymis and testicular abscess with possible destructions and subsequent fibrosis.

\section{CONCLUSION}

We recommend that acute epididymo-orchitis should be remembered as a sequel of $S$. pneumoniae bacteremia especially in adult males with immune suppression.

CONFLICT OF INTEREST: None to declare.

FINANCIAL INTEREST: None to declare.

\section{REFERENCES}

1. Musher DM, Alexandraki I, Graviss EA, et al. Bacteremic and non-bacteremic pneumococcal pneumonia. A prospective study. Medicine (Baltimore) 2000;79:210-221.

2. Vergara-López S, Pérez L, Corzo JE, Gómez-Mateos JM. Purulent pericarditis as a complication of bacteraemic pneumococcal pneumonia. Rev Clin Esp 2008;208:531.

3. Sexton DJ, Bartlett JG, Thorner AR. Invasive pneumococcal (Streptococcus pneumoniae) infections and bacteremia. Literature review current through. Uptodate 2012.

4. Krieger JN. Epididymitis, orchitis and related conditions. Sex Transm Dis 1984;11:173-181. 
5. Delavierre D. Orchi-epididymitis. Ann Urol (Paris) 2003;37:322-338.

6. Trojian TH, Lishnak TS, Heiman D. Epididymitis and orchitis: an overview. Am Fam Physician 2009;79:583-587.

7. Bansal N, Kaistha N, Chander J. Epididymoorchitis: an unusual manifestation of salmonellosis. J Microbiol Immunol Infect 2012;45:318-320.

8. Kashiwagi B, Okugi H, Morita T, Kato Y, Shibata Y, Yamanaka H. Acute epididymo-orchitis with abscess formation due to Pseudomonas aeruginosa: report of 3 cases. Hinyokika Kiyo 2000;46:915-918.

9. Papatsoris AG, Mpadra FA, Karamouzis MV, Frangides CY. Endemic brucellar epididymo-orchitis: a 10year experience. Int J Infect Dis 2002;6:309-313.

10. Suankwan U, Larbcharoensub N, Viseshsindh W, Wiratkapun C, Chalermsanyakorn P. A clinicopathologic study of tuberculous epididymo-orchitis in Thailand. Southeast Asian J Trop Med Public Health 2012;43:951958.

11. Nuorti JP, Butler JC, Farley MM, et al. Cigarette smoking and invasive pneumococcal disease. Active Bacterial Core Surveillance Team. N Engl J Med 2000;342:681-689.

12. Hamm B. Differential diagnosis of scrotal masses by ultrasound. Eur Radiol 1997;668-679

13. Pavlica P, Barozzi L. Imaging of the acute scrotum. Eur Radiol_2001;11:220-228.

14. Mäkelä E, Lahdes-Vasama $\mathrm{T}$, Ryymin $\mathrm{P}$, et al. Magnetic resonance imaging of acute scrotum. Scand J Surg 2011;100:7;196-201.

15. Street E, Joyce A, Wilson J. Clinical Effectiveness Group, British Association for Sexual Health and HIV, BASHH UK guideline for the management of epididymoorchitis 2010. Int J STD AIDS 2011;22:361-365.

\section{Citing this article}

Ibrahim TM, Letchumanan MN. Acute epididymoorchitis complicating Streptococcus pneumoniae bacteremia in an adult smoker and alcoholic with community acquired pneumonia. Int $\mathrm{J}$ Infect Microbiol 2012;1(2);80-83. 